\title{
Quantum dot loaded immunomicelles for tumor imaging
}

\author{
Aristarchos Papagiannaros, Jaydev Upponi, William Hartner, Dmitriy Mongayt, Tatyana Levchenko,
} Vladimir Torchilin

\begin{abstract}
Background: Optical imaging is a promising method for the detection of tumors in animals, with speed and minimal invasiveness. We have previously developed a lipid coated quantum dot system that doubles the fluorescence of PEG-grafted quantum dots at half the dose. Here, we describe a tumor-targeted near infrared imaging agent composed of cancer-specific monoclonal anti-nucleosome antibody 2C5, coupled to quantum dot (QD)-containing polymeric micelles, prepared from a polyethylene glycol/phosphatidylethanolamine (PEG-PE) conjugate. Its production is simple and involves no special equipment. Its imaging potential is great since the fluorescence intensity in the tumor is twofold that of non-targeted QD-loaded PEG-PE micelles at one hour after injection.
\end{abstract}

Methods: Para-nitrophenol-containing (5\%) PEG-PE quantum dot micelles were produced by the thin layer method. Following hydration, 2C5 antibody was attached to the PEG-PE micelles and the QD-micelles were purified using dialysis. 4T1 breast tumors were inoculated subcutaneously in the flank of the animals. A lung pseudometastatic B16F10 melanoma model was developed using tail vein injection. The contrast agents were injected via the tail vein and mice were depilated, anesthetized and imaged on a Kodak Image Station. Images were taken at one, two, and four hours and analyzed using a methodology that produces normalized signal-tonoise data. This allowed for the comparison between different subjects and time points. For the pseudometastatic model, lungs were removed and imaged ex vivo at one and twenty four hours.

Results: The contrast agent signal intensity at the tumor was double that of the passively targeted QD-micelles with equally fast and sharply contrasted images. With the side views of the animals only tumor is visible, while in the dorsal view internal organs including liver and kidney are visible. Ex vivo results demonstrated that the agent detects melanoma nodes in a lung pseudometastatic model after a 24 hours wash-out period, while at one hour, only a uniform signal is detected.

Conclusions: The targeted agent produces ultrabright tumor images and double the fluorescence intensity, as rapidly and at the same low dose as the passively targeted agents. It represents a development that may potentially serve to enhance early detection for metastases.

\section{Background}

Near infrared (NIR) imaging is a particularly promising method of imaging since it is not invasive, requires relatively simple and easy-to-use equipment, and can take place in real time. The detection limit can be as low as in other imaging modalities, and it is much less hazardous compared to radionuclide or magnetic resonance

\footnotetext{
* Correspondence: v.torchilin@neu.edu

Department of Pharmaceutical Sciences and Center for Pharmaceutical Biotechnology and Nanomedicine, 312 Mugar Life Sciences Building, Northeastern University, Boston MA 02115, USA
}

imaging as it does not make use of radioisotopes that have special handling and storage requirements, both for their use and their disposal [1]. It is also very versatile and affordable. Instruments are not as expensive or complicated as those for nuclear or magnetic imaging [2]. In the NIR, the light penetrates much further through the body compared to imaging in the visible part of the spectrum [3]. This 'absorbance window' allows for the visualization of various phenomena deep inside the body. Using targeted contrast agents, the fluorescence signal can be highly localized. Examples 
include the detection of the epidermal growth factor receptor [4] and vascular endothelial growth factor receptors in mice [5], or cathepsin metal-proteases in early pancreatic cancer [3]. Detailed and precise images of anatomical and functional aspects of animals can be produced using actively targeted fluorophores [6,7], but a high signal-to-noise ratio is difficult to achieve, since NIR fluorescence is typically scattered throughout the tissues of mice [8]. Thus, the need for highly fluorescent targeted nanoparticles that will allow precise optical imaging with a high signal-to-noise ratio and minimal invasiveness and using simple instrumentation remains.

Quantum dots (QD) are semiconductor nanocrystals made of inorganic materials, such as CdSe. They exhibit nanosized dimensions and low polydispersity [9] Their excitation and emission spectra depend on their size, so that different emission spectra can be produced essentially from the same materials simply by changing their size. This allows simultaneous imaging of different aspects of a pathological site [10] Their advantages for imaging include bright fluorescence, excellent photo-stability, and variety in possible emission spectra [11-13]. They are ideal for optical imaging and considered reasonably safe, provided that a stable coating is applied during their manufacture $[11,14]$. Their applications include cell labeling [15], cell trafficking studies [16], sentinel lymph node imaging [17], lymphatic imaging [18], detection of apoptosis [19], tumor detection, and brain imaging [20]. NIR-emitting QD that exhibit a high molar excitation coefficient are particularly suited for the in vivo whole body imaging. They can allow the detection and tracking of single cells throughout the body [21]. Since their surface properties determine their biodistribution, various modifications of QD have been tested to achieve long circulation times and either passive or active targeting to areas of interest [22,23].

Lipid-core micelles are a versatile system for the administration of drugs, DNA or imaging agents [24] and they have proven to be a safe and highly biocompatible system [25-27]. They are composed mainly of amphiphilic block-copolymers composed of soluble blocks, such as polyethylene glycol (PEG), and insoluble lipid blocks, such as phosphatidylethanolamine (PE). They self-assemble when the concentration of the amphiphilic copolymers is above their critical micellar concentration, exhibit both high stability and excellent biocompatibility, and are very stable as long as their concentration in the plasma is higher than their critical micellar concentration. They have been used extensively for the delivery of potent but poorly soluble drugs for cancer therapy, like paclitaxel, or meso-tetraphenylporphin in photodynamic therapy $[28,29]$. They have also been used as contrast agent carriers for tumor imaging, such as QD carriers in whole body optical imaging, as carriers of super-paramagnetic nanoparticles for magnetic resonance imaging and with radionuclides, such as ${ }^{111}$ In, for gamma imaging studies [25].

Recently, we introduced a NIR contrast agent for whole body imaging composed of QD-loaded PEG-PE micelles (QD-Mic). The advantages of this formulation compared to commercially available formulations include the rapid accumulation of the agent at the tumor site (one hour compared to four hours for the commercial formulation) and sharply contrasted images. Using a novel quantification method for planar imaging, we were able to determine that QD-Mic doubled the signal at the tumor site with half the dose compared to passively targeted QDMic [30]. Thus, the discrimination of tumors and internal organs was possible. Here, we present the next step in the development of QD-Mic, by actively targeting QD-Mic to cancer cells. This was possible by the additional modification of the PEG-PE coat of QD-Mic, to which a broad variety of targeting ligands can be attached. In this study, we used QD-Mic modification with the monoclonal antibody $(\mathrm{mAb}) 2 \mathrm{C} 5$, which has a nucleosome-restricted specificity and recognizes a variety of cancer cells via cancer cell surface-bound nucleosomes released from the apoptotically dying neighboring cancer cells [31]. mAb 2C5-targeted micelles were shown to accumulate specifically in tumors and increase the anti-tumor effect of micelle-incorporated drugs [28]. Nucleosomes are abundant on the surface of all uncontrollably proliferative cancer cells. Recent reports from our lab demonstrate the use of mAb 2C5-labeled micelles or liposomes, both to established tumors and metastasis, including B16 melanoma tumors [32-34]. Such immunomicelles can attach to cancer cells even when the tumor is small, and may serve as a vehicle for the early detection and treatment of cancer micrometastasis [25]. Early detection (and treatment) of metastasis is of great importance for the cure of cancer, since the mortality is usually due to the development metastatic cancers and not to the primary tumor $[35,36]$.

In this paper, we describe the development of 2C5-targeted QD-loaded PEG-PE-based immunomicelles that can detect small tumor sites.

\section{Methods}

\section{Micelle characterization and stability}

Micelle size was determined by the dynamic light scattering (Beckman Coulter N4 Plus, USA).

\section{Preparation of QD-Mic}

Near infrared emitting CdSe QD (Qdots 800, Invitrogen, USA) were incorporated in micelles as previously described $[25,37,38]$. Briefly, a QD suspension in decane was mixed with a four-fold volume of a 1:3 isopropanol/ methanol mixture and centrifuged at $3500 \mathrm{rpm}$ for $5 \mathrm{~min}$. The supernatant was discarded and the QD pellet was 
re-suspended in chloroform. For micelle production, $2.7 \mu$ moles of PEG2000-PE [N-carbonyl-methoxy-poly(ethyleneglycol-2000)-1,2-distearoyl-3-phospho-ethanolamine sodium salt] containing a $5 \%$ molar ratio of the reactive para-nitrophenylcarbonyl(pNP)-PEG2000-PE was mixed with 20 pmoles of QD in chloroform, and solvents were evaporated under vacuum. The system was freezedried overnight and, when necessary, hydrated in phosphate buffered saline (PBS), $\mathrm{pH}$ 7.4, or citrate buffer, $\mathrm{pH}$ 5.0. Micelle size was determined.

\section{Conjugation of 2C5 antibodies to QD-Mic}

The PEG-PE/QD mixture was hydrated in $200 \mu \mathrm{l}$ of the citrate buffer, $\mathrm{pH}$ 5.0, with vortexing. After an equilibration period of 1 hour, the micelles were incubated in a 3-fold excess borate buffer, $\mathrm{pH}$ 9.3, and mixed with a 2 -fold excess of $2 \mathrm{C} 5$ antibody ( $339 \mu \mathrm{l}$ of a $2.84 \mathrm{mg} / \mathrm{ml}$ solution). They were then dialyzed (cut-off value $\mathrm{MW}$ $250,000)$ overnight against PBS, pH 7.4 at $4^{\circ} \mathrm{C}$.

\section{Tumor inoculation in mice}

Female Balb/c mice, 6-8 weeks old, (Charles River Laboratories, Wilmington, MA) were inoculated with tumors following a protocol approved by the Northeastern University Institutional Animal Care and Use Committee in accordance with the 'Principles of Laboratory Animal Care' (NIH publication No. 85-23, revised in 1996). The $4 \mathrm{~T} 1$ murine breast cancer cells were grown in Dulbecco Modified Eagle's Medium (DMEM) supplemented with $10 \%$ fetal calf serum. Cells $\left(1.5 \times 10^{5}\right.$ per mouse) were suspended in $150 \mu \mathrm{l}$ of PBS and injected subcutaneously in the right flank. Tumors developed within two weeks after the injection. The animals had free access to food and water.

\section{Pseudometastatic melanoma model}

B16F10 cells were grown in DMEM supplemented with antibiotics and $10 \%$ fetal calf serum. Cells $\left(8 \times 10^{5}\right.$ per mouse) were injected in the tail vein. In approximately two weeks, cancer cell nodes developed in the lungs of the animals. Control animals were sacrificed by carbon dioxide euthanasia, lungs removed, washed in saline, and the melanoma nodes were counted. Ex vivo white field photographs of the lungs were taken using the Kodak Image Station In Vivo FX (Carestestream Health, USA). Cancer cell nodes were easily recognized as the black spots on the lung surface.

\section{Near Infrared mouse imaging}

Contrast agents, 40 pmoles/mouse [11] were injected via the tail vein. For the pseudometastasic model, 1/10 of the above dose was injected. After the administration of the contrast agents, mice were anesthetized (ketamine/ xylazine, i.p.) and depilated using a hair removal cream
(Nair, USA) prior to tumor observation. Images were taken with a Kodak Image Station (filters: excitation $710 \mathrm{~nm}$, emission $790 \mathrm{~nm}$ ) at different angles. At the end of the experiment, mice were sacrificed with $\mathrm{CO}_{2}$, skin removed to avoid its scattering of the fluorescence, and images were retaken to precisely localize the internal organs. All images were analyzed using the Kodak Image Analysis software or the ImageJ (NIH, USA). The regions of interest (ROI) were determined using threshold analysis by comparing the whole body images with the images after the skin was removed. The mean pixel intensity, the background signal and the autofluorescence of the animal was extracted at each time point. The mean pixel intensity at the ROI was expressed as an absolute number by comparison with the scattered light (background noise from the internal organs near the ROI), and all images were normalized using a ROI over the hip of the mouse to compensate for differences between the NIR images of the different animals and time points that result from differences in the overall image luminosity.

\section{Detection of cancer cell nodes in the pseudometastic model}

Contrast agents were injected as previously described. At one hour and twenty four hours after the injection of the contrast agent, animals were sacrificed and lungs removed. Images of the removed organs were taken as before, both in white field and in near infrared.

\section{Results}

\section{Micelle size}

The size of the 2C5 QD-Mic was $21.0 \pm 6.7 \mathrm{~nm}$ and QD-M $17.6 \pm 1.2 \mathrm{~nm}$. Micelles were stable at $4^{\circ} \mathrm{C}$ for at least fifteen days.

\section{Signal-to-noise ratio}

Figure 1 shows the signal-to-noise for the 2C5-QD-Mic at the area over the tumor over 4 hours. The signal-to-noise is high within the first hour and remains practically unchanged for the duration of the imaging. One hour after the injection, the signal-to-noise was $47.1 \pm 3.1$, while at the end of imaging it was $49.8 \pm 2.7$. This signal was double that of the non-targeted QD-Mic and almost four times higher that of the commercially available formulation of PEG grafted quantum dots (Qtracker, Invitrogen, USA) administered at double the dose published earlier [30].

\section{Biodistribution of the 2C5-QD-Mic using image analysis}

Figure 2 shows the distribution of the NIR signal to the various tissues. The methodolody, as published earlier, involves the generation of weighted signal-to-noise data for the duration of the imaging experiment. The signal-to-noise is comparable among the different time 


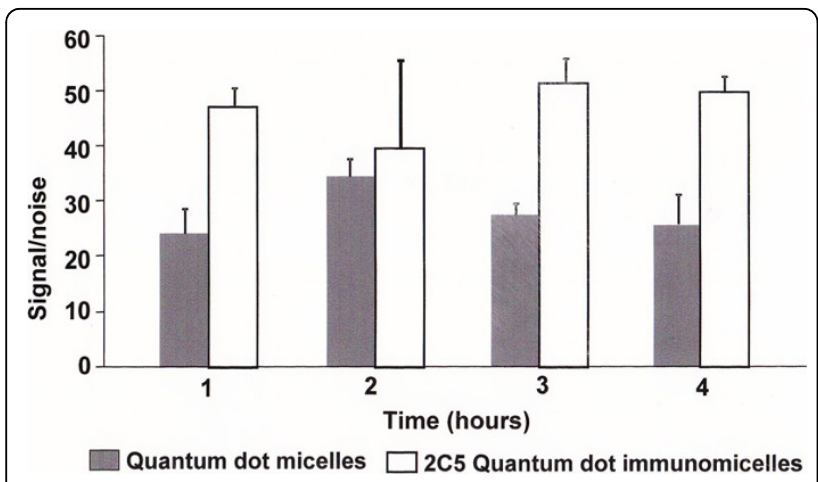

Figure 1 Normalized signal-to-noise ratio for the fluorescence of the tumor. Data show the ratio of the tumor fluorescence vs. the background in the area around the tumor for 2C5 modified QDMic and for QD-Mic without antibody. Error bars represent standard deviation, $\mathrm{N}=5$.

points, as it represents normalized values with reference to the intrinsic fluorescence of the animal. This analysis allowed us to express and quantify the imaging effect of the contrast agent in vivo [39]. The highest signal was detected in the tumor area $(47.2 \pm 3.1$ at one hour after the injection) and the kidney (51.4 \pm 25.2). The rest of the organs exhibited a lower signal, the lowest of which was from the liver with the signal intensity of $4.97 \pm 0.26$. This pattern remained unchanged throughout the imaging period, so that at four hours after the administration of the contrast agent, the signal in the tumor area was $49.8 \pm 2.7$. The liver again exhibited the lowest signal of $5.64 \pm 0.82$. With the exception of the kidney and spleen, the actively targeted 2C5 QD-Mic persisted in the tumor area with lower affinity for other organs.

\section{Composite images of mice injected with 2C5-QD-Mic}

Figure 3 shows composite NIR images of two mice injected with 2C5-QD-Mic one hour after the injection,

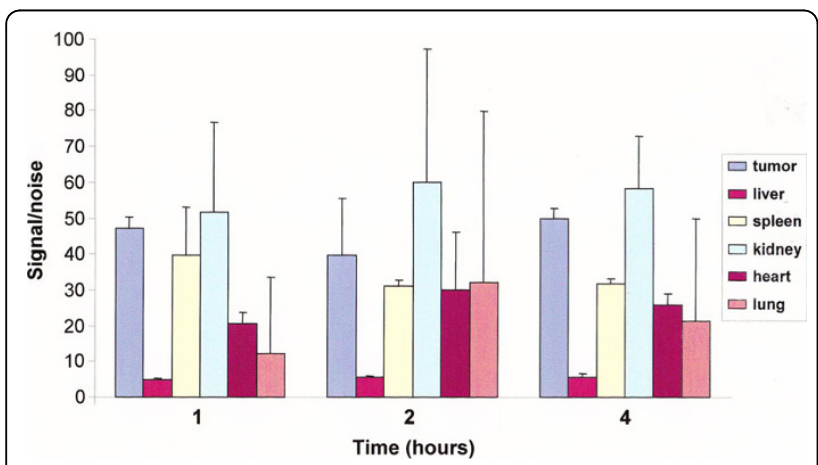

Figure 2 Quantification of the biodistribution of 2C5 QD-M in animals. Units represent net light intensity in the near infrared region weighted vs. the autofluorescence of the mouse. Error bars represent standard deviation, $\mathrm{N}=5$. superimposed over a white field image. The signal is visible only from the tumor area, indicated by the arrow. Some signal was detected from hairs that were not completely removed. The histograms of the pixel values (Figure 3) verified this conclusion. Pixel values for the tumor area had the highest values compared to the rest of the animal body. For instance, the mean value in the non-tumor ROI is $42.8 \pm 23.5$, and $62.2 \pm$ 16.1 in the tumor area. It is of particular interest that the pixel distribution is much narrower in the tumor ROI. The high slope of the pixel value distribution allowed the tumor to be identified clearly.

\section{Composite ex vivo images of lungs from mice injected with 2C5-QD-Mic in a melanoma pseudo-metastatic model}

In Figure 4, B16F10 melanoma cells appeared black, while normal cells are white, since Balb/c mice are albino. The whole body images from control mice injected with B16F10 melanoma cells, but not the 2C5QD-Mic represents the intrinsic fluorescence of the organ and showed a low level of uniform NIR fluorescence. The cancer cell clusters were clearly identifiable by their black color. The background fluorescence was uniformly distributed over the lungs. A large number of cancer cell nodes could be seen in the white field photograph. One hour after the injection of the 2C5-QD-Mic, the NIR fluorescence was still diffuse over the entire lungs with a pattern similar to the background fluorescence but with a slightly higher intensity. At 24 hours after injection, NIR fluorescence was much less uniform. Signal was concentrated mainly at areas near the small clusters of cancer cells and in the periphery of the lungs, pinpointing the smaller cancer cell clusters.

\section{Discussion}

The need for efficient contrast agents surfaced with the development of practical and easy-to-use instruments for NIR whole body imaging. Lipid-based coatings for QD were proposed by us and by other labs involves encapsulation of one quantum dot within a lipid envelope $[37,38,40,41]$. Our approach described aimed to alter the biodistribution profile of QD to allow for effective and relatively rapid imaging of tumor sites. With this in mind, we have developed a contrast agent that actively targets tumor sites and results in a high signal-to-noise ratio. This formulation represents a significant improvement over both the commercially available pegylated QD such as the Qtracker ${ }^{\circ}$, and passively targeted QDcontaining PEG-PE micelles earlier described [30], since it allows for rapid imaging using a low dose of QD (half that of the Qtracker ${ }^{\circ}$ ) and with a two-fold enhancement of image intensity compared to non-targeted QD-Mic at the same QD dose. The encapsulation of QD in a PEG- 


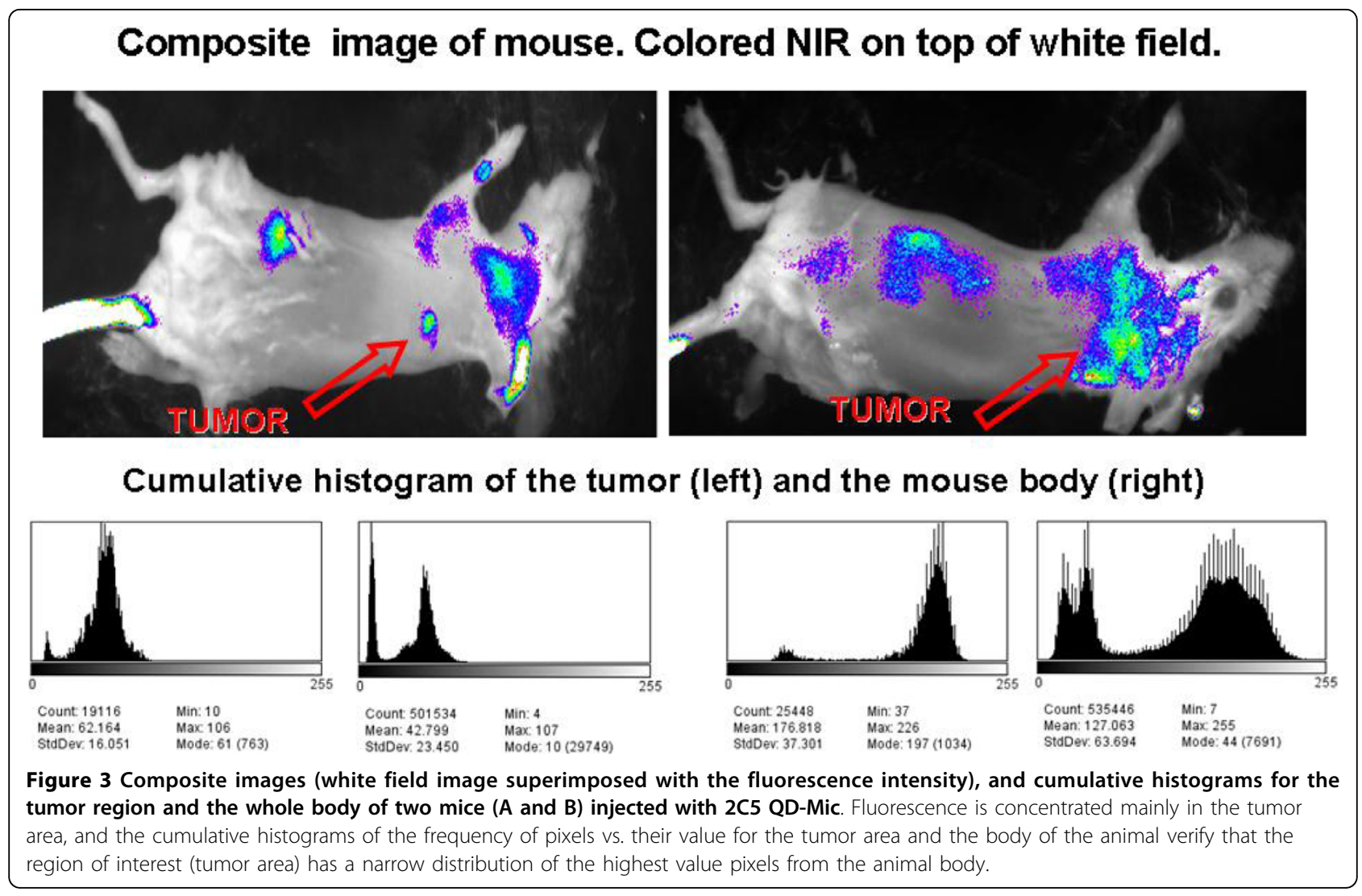

PE envelope allows for the easy attachment of antibodies and other ligands without using complex surface chemistry $[40,41]$. The attachment of antibodies was performed via the micelle-incorporated reactive pNP-PEG-PE component, which is simple and straight-forward compared to the use of an avidin-biotin linker that exposes the complex to opsonization by blood proteins and may decrease its circulation time [42]. This method also offers advantages such as simplicity and high stability over systems composed of multiple cores in a complex lipid system [43].

Previously, we showed that the encapsulation of QD into the PEG-PE envelope increases the signal of QD in the tumor area compared to unmodified "native" QD, decreases the necessary imaging time from four hours to one hour, and allows for a decrease of the equivalent dose of the imaging agent by one half. The attachment of the anti-tumor 2C5 antibody further doubles the signal by bringing more contrast agent into the tumor, similar to earlier demonstrated enhanced imaging by 2C5-targeted contrast agents with other imaging modalities [44]. This targeting allowed us to image the tumor site with a superior signal especially to that of the liver. This increase in the signal of 2C5 QD-M was also reported in a variety of earlier papers from our lab using the anti-nucleosome 2C5 anticancer antibody which is generic to all uncontrollably proliferating cell lines [29]. The effect of targeting has been demonstrated in various systems, including doxorubicin liposomes modified with the post-insertion method [33] and micelles [45] and using different imaging modalities such as MRI [44] or gamma imaging [46].

Antibodies are well known agents that increase the accumulation at sites overexpressing tumor antigens $[47,48]$ The attachment of antibodies permits the targeting and visualization of surface antigens in low concentrations [49] and especially micrometastasis [50]. High affinity antibodies saturate the surface of the tumor and it becomes difficult to access the tumor mass, thus the enhanced permeation effect can become critical [51] and in fact, this effect, was recently modeled stochastically [52-55]. Nanoparticles accumulate mainly through passive targeting while the tumor localization of the nanoparticles is influenced by the antibody [56]. The antibodies therefore are essential for the specificity of the cell targeting while the passive accumulation is the main force for the biodistribution of the nanoparticles to the solid tumor [57]. This is a proof of the versatility of our imaging methodology, that produces data as normalized signal to noise, which quantify the imaging effect (contrast) rather than simply the amount of the nanocarrier present at the tumor site. This was also 

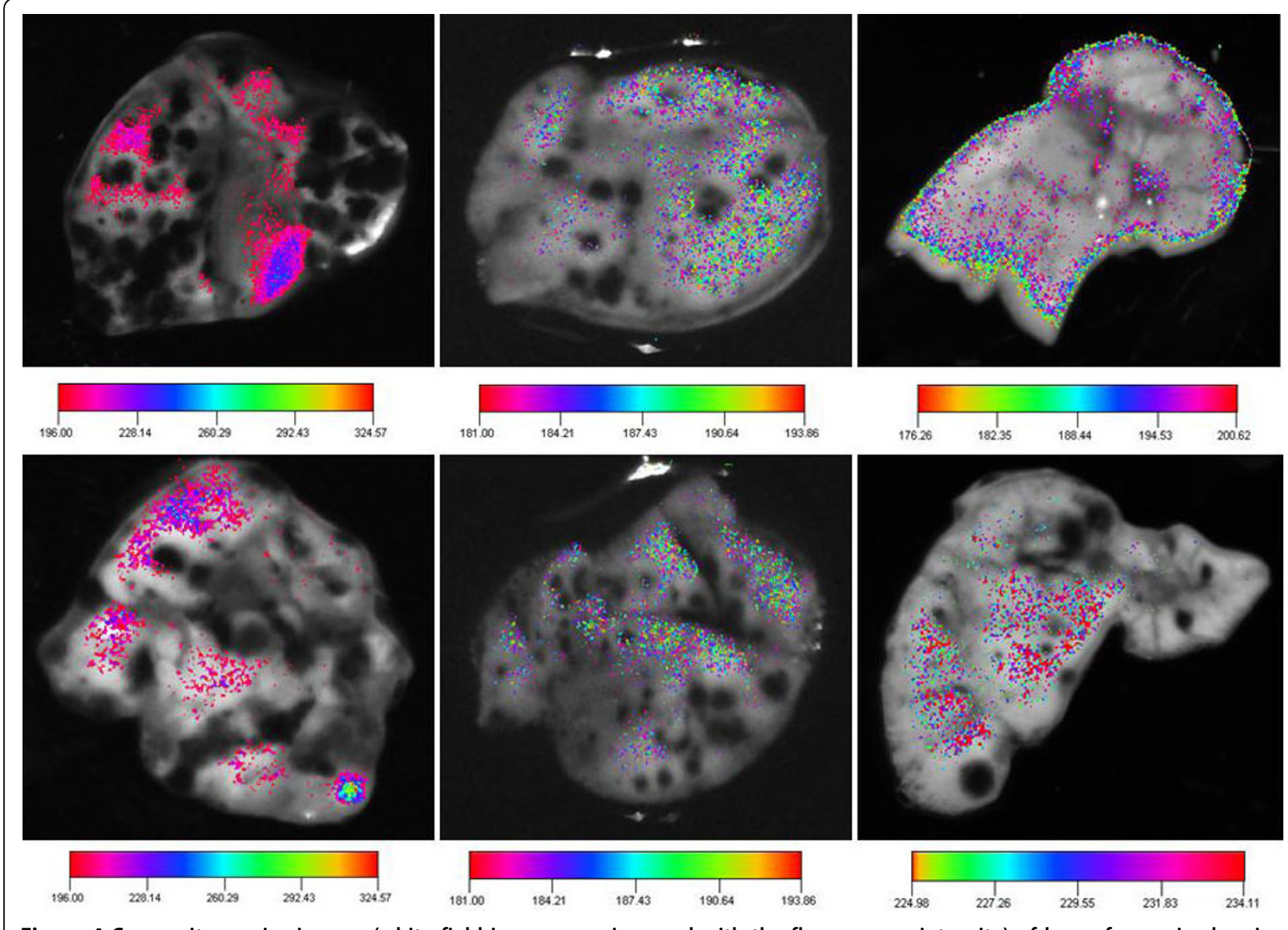

Figure 4 Composite ex vivo images (white field image superimposed with the fluorescence intensity) of lungs from mice bearing metastatic B16F-10 lung melanoma tumor. Left: two mice not injected with 2C5 QD-Mic. We see the clusters of melanoma cells (black) in the lungs (white) and a low level of near infrared fluorescence. Center: two mice injected with 2C5 QD-Mic at one hour after the injection. There is a higher level of near infrared fluorescence from the lungs. Right: two mice injected with 2C5 QD-Mic at twenty-four hours after the injection. The fluorescence originates mainly from sites around the melanoma clusters allows detection of the metastatic sites.

done by other groups, expressing the targeting effect as a two-fold increase over the passive accumulation, using radioactivity and calculating the ratio of the tumor vs. the hip muscle [58].

The use of lipid-based nanoparticles for delivery of QD has recently gained a lot of attention. A hybrid QD/cationic liposome system was recently assembled [59]. This system significantly enhanced the delivery of QD in tridimensional cell culture systems, due mainly to the effect of the positive charge of the nanoparticles. Another system used conventional immunoliposomes [60] with QD attached via PEG spacers at the surface of the nanoparticles. This latter system offers bright images due to the presence of a large number of fluorophores per nanoparticle. The simplicity of the 2C5-QD-Mic may provide an advantage. 2C5-QD-Mic represent an improvement over QD directly conjugated to an antibody, where the QD antibody complex lacks the steric protection provided by PEG coating [61].
The greater efficiency provided by the higher signal within a shorter time should be of great benefit for cancer imaging. Recent studies have shown that QD toxicity may be low enough for systemic administration [62]. The small number of studies on the use of QD for the detection of metastases [62-64] may be attributed to the low sensitivity of the optical imaging and the intense scattering of the light that makes pinpointing of the source and estimation of the size of small tumors difficult. The clear benefit of our formulation is its high affinity towards cancer cells introduced by the $2 \mathrm{C} 5$ antibody $[28,65]$. Although the period of 24 hours for the detection of metastases is longer than that of the imaging of a larger tumor (one hour), this is due to the need to allow the contrast agent long enough time to circulate and attach in sufficient quantity to the cancer cells in loci containing small numbers of such cells. This observation also implies that both passive targeting and antibody-mediated attachment to the tumor are involved in the accumulation of the targeted contrast 
agent at the tumor site. The lung tissues are first saturated with the contrast agent and the attachment of 2C5-QDMic to cancer loci follows together with the elimination of the unbound contrast agents from the lungs as a whole. The insufficient staining of bigger cancer masses may be explained by an absence of 2C5-QD-Mic penetration of the tumor cells.

The use of NIR optical imaging for the detection of metastases has gained more attention recently and is a useful modality for the intra-operative detection of lymph node metastases [66]. By administering quantum dots before the operation the lymph nodes can be visualized during the operation for the removal of a breast cancer and reduce the probability for the development of metastases. Optical methodology has obvious advantages, due to the simplicity of the instruments involved, for use in the operating room [67-70]. This imaging effect is in accordance with observations that the main thrust for tumor accumulation is the EPR effect while the antibody plays a helping role, which is limited to cell penetration, especially by large lipid nanoparticles such as liposomes. In fact, in many cases the antibodies stain only the periphery of the cell cluster and are unable to penetrate deeply inside the tumor mass. The net effect is a resultant drainage of the nanoparticles from the tumor $[52,56]$.

However, as a modality for the detection of metastasis, near infrared imaging has limits, because of the low sensitivity of the method and the inability to detect cancer clusters in whole body imaging. Recent developments involving the quantification of the signal and the detection of fluorescence deep inside the body may make this type of imaging possible and useful [71]. Although the time necessary for imaging may be longer with QD-Mic than with some radioimaging contrasts, the benefits of using non-radiation emitting agents remain important. As evident from our data, there is a sufficient difference from the background signal of the healthy part of the lungs to permit localization of cancer cells within healthy organs. Our system may be useful to allow the detection of a signal from remote sources throughout the body and permit visualization of small clusters of cancer cells before they develop into apparent tumors [72], especially if coupled with an advanced imaging modality that captures in vivo images in real time [73,74].

\section{Conclusion}

In summary, 2C5-QD-Mic allow imaging as equally rapid as the non-targeted micelles and produce twice the signal at the same dose. Their production is easy and requires no special equipment. With the development of advanced imaging instruments that allow signal detection deep within the body, their potential usefulness can be expected to be significantly enhanced.

\section{Acknowledgements}

This work was supported by NIH grant RO1 EB001961 to V.P. Torchilin.

\section{Authors' contributions}

AP, Conception and design of the contrast agents and the image analysis methodology, execution of experiments and draft of the paper, JU contrast agent preparation and animal imaging, DM. 2C5 antibody conjugation, WH animal experiments, $\mathrm{TL}$ and $\mathrm{VT}$ conception and design of the study. All authors read and approved the manuscript.

\section{Competing interests}

The authors declare that they have no competing interests.

Received: 14 December 2009 Accepted: 18 October 2010 Published: 18 October 2010

\section{References}

1. Rao J, Dragulescu-Andrasi A, Yao H: Fluorescence imaging in vivo: recent advances. Curr Opin Biotechnol 2007, 18:17-25.

2. Nyk M, Kumar R, Ohulchanskyy TY, Bergey EJ, Prasad PN: High contrast in vitro and in vivo photoluminescence bioimaging using near infrared to near infrared up-conversion in $\mathrm{Tm} 3+$ and $\mathrm{Yb3}+$ doped fluoride nanophosphors. Nano Lett 2008, 8:3834-3838.

3. von Burstin J, Eser S, Seidler B, Meining A, Bajbouj M, Mages J, Lang R, Kind AJ, Schnieke AE, Schmid RM, Schneider G, Saur D: Highly sensitive detection of early-stage pancreatic cancer by multimodal near-infrared molecular imaging in living mice. Int J Cancer 2008, 123:2138-2147.

4. Ke S, Wen X, Gurfinkel M, Charnsangavej C, Wallace S, Sevick-Muraca EM, Li C: Near-infrared optical imaging of epidermal growth factor receptor in breast cancer xenografts. Cancer Res 2003, 63:7870-7875.

5. Backer MV, Levashova Z, Patel V, Jehning BT, Claffey K, Blankenberg FG, Backer JM: Molecular imaging of VEGF receptors in angiogenic vasculature with single-chain VEGF-based probes. Nat Med 2007, 13:504-509.

6. Raman V, Artemov D, Pathak AP, Winnard PT Jr, McNutt S, Yudina A, Bogdanov A Jr, Bhujwalla ZM: Characterizing vascular parameters in hypoxic regions: a combined magnetic resonance and optical imaging study of a human prostate cancer model. Cancer Res 2006, 66:9929-9936

7. Gao Y, Kilfoil ML: Accurate detection and complete tracking of large populations of features in three dimensions. Opt Express 2009, 17:4685-4704.

8. Adams KE, Ke S, Kwon S, Liang F, Fan Z, Lu Y, Hirschi K, Mawad ME, Barry MA, Sevick-Muraca EM: Comparison of visible and near-infrared wavelength-excitable fluorescent dyes for molecular imaging of cancer. J Biomed Opt 2007, 12:024017.

9. Biju V, Itoh T, Anas A, Sujith A, Ishikawa M: Semiconductor quantum dots and metal nanoparticles: syntheses, optical properties, and biological applications. Anal Bioanal Chem 2008, 391:2469-2495.

10. Xia Z, Xing Y, So MK, Koh AL, Sinclair R, Rao J: Multiplex detection of protease activity with quantum dot nanosensors prepared by inteinmediated specific bioconjugation. Anal Chem 2008, 80:8649-8655.

11. Gao X, Yang L, Petros JA, Marshall FF, Simons JW, Nie S: In vivo molecular and cellular imaging with quantum dots. Curr Opin Biotechnol 2005, 16:63-72.

12. Xing $Y$, Chaudry Q, Shen C, Kong KY, Zhau HE, Chung LW, Petros JA O'Regan RM, Yezhelyev MV, Simons JW, Wang MD, Nie S: Bioconjugated quantum dots for multiplexed and quantitative immunohistochemistry. Nat Protoc 2007, 2:1152-1165.

13. Tian Z, Chen G, Li X, Liang H, Li Y, Zhang Z, Tian Y: Autofluorescence-free in vivo multicolor imaging using upconversion fluoride nanocrystals. Lasers Med Sci 2009.

14. Wang L, Nagesha DK, Selvarasah S, Dokmeci MR, Carrier RL: Toxicity of CdSe Nanoparticles in Caco-2 Cell Cultures. J Nanobiotechnology 2008, 6:11.

15. Jaiswal JK, Goldman ER, Mattoussi H, Simon SM: Use of quantum dots for live cell imaging. Nat Methods 2004, 1:73-78.

16. Jaiswal JK, Simon SM: Optical monitoring of single cells using quantum dots. Methods Mol Biol 2007, 374:93-104.

17. Kim S, Lim YT, Soltesz EG, De Grand AM, Lee J, Nakayama A, Parker JA, Mihaljevic T, Laurence RG, Dor DM, Cohn LH, Bawendi MG, Frangioni JV: 
Near-infrared fluorescent type II quantum dots for sentinel lymph node mapping. Nat Biotechnol 2004, 22:93-97.

18. Hama Y, Koyama Y, Urano Y, Choyke PL, Kobayashi H: Simultaneous twocolor spectral fluorescence lymphangiography with near infrared quantum dots to map two lymphatic flows from the breast and the upper extremity. Breast Cancer Res Treat 2007, 103:23-28.

19. van Tilborg GA, Mulder WJ, Chin PT, Storm G, Reutelingsperger CP, Nicolay K, Strijkers GJ: Annexin A5-conjugated quantum dots with a paramagnetic lipidic coating for the multimodal detection of apoptotic cells. Bioconjug Chem 2006, 17:865-868.

20. Wang J, Yong WH, Sun Y, Vernier PT, Koeffler HP, Gundersen MA, Marcu L: Receptor-targeted quantum dots: fluorescent probes for brain tumor diagnosis. J Biomed Opt 2007, 12:044021.

21. Xing Y, Smith AM, Agrawal A, Ruan G, Nie S: Molecular profiling of single cancer cells and clinical tissue specimens with semiconductor quantum dots. Int J Nanomedicine 2006, 1:473-481.

22. Jiang W, Papa E, Fischer H, Mardyani S, Chan WC: Semiconductor quantum dots as contrast agents for whole animal imaging. Trends Biotechnol 2004, 22:607-609.

23. Freeman R, Finder T, Bahshi L, Willner I: Beta-cyclodextrin-modified CdSe/ $\mathrm{ZnS}$ quantum dots for sensing and chiroselective analysis. Nano Lett 2009, 9:2073-2076.

24. Torchilin VP: Lipid-core micelles for targeted drug delivery. Curr Drug Deliv 2005, 2:319-327.

25. Torchilin VP: Targeted pharmaceutical nanocarriers for cancer therapy and imaging. Aaps J 2007, 9:E128-147.

26. Torchilin VP: Micellar nanocarriers: pharmaceutical perspectives. Pharm Res 2007, 24:1-16.

27. Torchilin V: Multifunctional and stimuli-sensitive pharmaceutical nanocarriers. Eur J Pharm Biopharm 2009, 71:431-444.

28. Torchilin VP, Lukyanov AN, Gao Z, Papahadjopoulos-Sternberg B: Immunomicelles: targeted pharmaceutical carriers for poorly soluble drugs. Proc Natl Acad Sci USA 2003, 100:6039-6044.

29. Roby A, Erdogan S, Torchilin VP: Enhanced In Vivo Antitumor Efficacy of Poorly Soluble PDT Agent, Meso-Tetraphenylporphine, in PEG-PE-Based Tumor-Targeted Immunomicelles. Cancer Biol Ther 2007, 6.

30. Papagiannaros A, Levchenko T, Hartner W, Mongayt D, Torchilin V: Quantum dots encapsulated in phospholipid micelles for imaging and quantification of tumors in the near-infrared region. Nanomedicine 2009, 5:216-224.

31. lakoubov LZ, Torchilin VP: Nucleosome-releasing treatment makes surviving tumor cells better targets for nucleosome-specific anticancer antibodies. Cancer Detect Prev 1998, 22:470-475.

32. Elbayoumi TA, Torchilin VP: Tumor-specific anti-nucleosome antibody improves therapeutic efficacy of doxorubicin-loaded long-circulating liposomes against primary and metastatic tumor in mice. Mol Pharm 2009, 6:246-254

33. ElBayoumi TA, Torchilin VP: Tumor-targeted nanomedicines: enhanced antitumor efficacy in vivo of doxorubicin-loaded, long-circulating liposomes modified with cancer-specific monoclonal antibody. Clin Cancer Res 2009, 15:1973-1980.

34. Skidan I, Dholakia P, Torchilin V: Photodynamic therapy of experimental B16 melanoma in mice with tumor-targeted 5,10,15,20tetraphenylporphin-loaded PEG-PE micelles. J Drug Target 2008, 16:486-493.

35. Riethdorf S, Wikman H, Pantel K: Review: Biological relevance of disseminated tumor cells in cancer patients. Int I Cancer 2008, 123:1991-2006

36. Wikman $\mathrm{H}$, Vessella $\mathrm{R}$, Pantel $\mathrm{K}$ : Cancer micrometastasis and tumour dormancy. Apmis 2008, 116:754-770.

37. Dubertret B, Skourides $P$, Norris DJ, Noireaux V, Brivanlou AH, Libchaber A: In vivo imaging of quantum dots encapsulated in phospholipid micelles. Science 2002, 298:1759-1762.

38. Stroh M, Zimmer JP, Duda DG, Levchenko TS, Cohen KS, Brown EB, Scadden DT, Torchilin VP, Bawendi MG, Fukumura D, Jain RK: Quantum dots spectrally distinguish multiple species within the tumor milieu in vivo. Nat Med 2005, 11:678-682.

39. Papagiannaros A, Kale A, Levchenko TS, Mongayt D, Hartner WC, Torchilin VP: Near infrared planar tumor imaging and quantification using nanosized Alexa 750-labeled phospholipid micelles. Int J Nanomedicine 2009, 4:123-131.
40. Carion O, Mahler B, Pons T, Dubertret B: Synthesis, encapsulation, purification and coupling of single quantum dots in phospholipid micelles for their use in cellular and in vivo imaging. Nat Protoc 2007, 2:2383-2390.

41. Dubois F, Mahler B, Dubertret B, Doris E, Mioskowski C: A versatile strategy for quantum dot ligand exchange. J Am Chem Soc 2007, 129:482-483.

42. Wu X, Liu H, Liu J, Haley KN, Treadway JA, Larson JP, Ge N, Peale F, Bruchez MP: Immunofluorescent labeling of cancer marker Her2 and other cellular targets with semiconductor quantum dots. Nat Biotechnol 2003, 21:41-46.

43. Park JH, von Maltzahn G, Ruoslahti E, Bhatia SN, Sailor MJ: Micellar hybrid nanoparticles for simultaneous magnetofluorescent imaging and drug delivery. Angew Chem Int Ed Engl 2008, 47:7284-7288.

44. Erdogan S, Medarova ZO, Roby A, Moore A, Torchilin VP: Enhanced tumor MR imaging with gadolinium-loaded polychelating polymer-containing tumor-targeted liposomes. J Magn Reson Imaging 2008, 27:574-580.

45. Musacchio T, Laquintana V, Latrofa A, Trapani G, Torchilin VP: PEG-PE micelles loaded with paclitaxel and surface-modified by a PBR-ligand: synergistic anticancer effect. Mol Pharm 2009, 6:468-479.

46. Elbayoumi TA, Torchilin VP: Enhanced accumulation of long-circulating liposomes modified with the nucleosome-specific monoclonal antibody 2C5 in various tumours in mice: gamma-imaging studies. Eur J Nucl Med Mol Imaging 2006, 33:1196-1205.

47. Adams GP, Schier R, Marshall K, Wolf EJ, McCall AM, Marks JD, Weiner LM: Increased affinity leads to improved selective tumor delivery of singlechain Fv antibodies. Cancer Res 1998, 58:485-490.

48. Baselga J, Norton L, Albanell J, Kim YM, Mendelsohn J: Recombinant humanized anti-HER2 antibody (Herceptin) enhances the antitumor activity of paclitaxel and doxorubicin against HER2/neu overexpressing human breast cancer xenografts. Cancer Res 1998, 58:2825-2831.

49. Howarth M, Liu W, Puthenveetil S, Zheng Y, Marshall LF, Schmidt MM, Wittrup KD, Bawendi MG, Ting AY: Monovalent, reduced-size quantum dots for imaging receptors on living cells. Nat Methods 2008, 5:397-399.

50. Chang MY, Seideman J, Sofou S: Enhanced loading efficiency and retention of 225Ac in rigid liposomes for potential targeted therapy of micrometastases. Bioconjug Chem 2008, 19:1274-1282.

51. Adams GP, Schier R, McCall AM, Simmons HH, Horak EM, Alpaugh RK, Marks JD, Weiner LM: High affinity restricts the localization and tumor penetration of single-chain fv antibody molecules. Cancer Res 2001, 61:4750-4755.

52. Ackerman ME, Pawlowski D, Wittrup KD: Effect of antigen turnover rate and expression level on antibody penetration into tumor spheroids. Mol Cancer Ther 2008, 7:2233-2240.

53. Thurber GM, Schmidt MM, Wittrup KD: Antibody tumor penetration: transport opposed by systemic and antigen-mediated clearance. Adv Drug Deliv Rev 2008, 60:1421-1434.

54. Thurber GM, Schmidt MM, Wittrup KD: Factors determining antibody distribution in tumors. Trends Pharmacol Sci 2008, 29:57-61.

55. Schmidt MM, Wittrup KD: A modeling analysis of the effects of molecular size and binding affinity on tumor targeting. Mol Cancer Ther 2009, 8:2861-2871.

56. Thurber GM, Wittrup KD: Quantitative spatiotemporal analysis of antibody fragment diffusion and endocytic consumption in tumor spheroids. Cancer Res 2008, 68:3334-3341.

57. Heidel JD, Davis ME: Clinical Developments in Nanotechnology for Cancer Therapy. Pharm Res.

58. Kirpotin DB, Drummond DC, Shao Y, Shalaby MR, Hong K, Nielsen UB, Marks JD, Benz CC, Park JW: Antibody targeting of long-circulating lipidic nanoparticles does not increase tumor localization but does increase internalization in animal models. Cancer Res 2006, 66:6732-6740.

59. Al-Jamal WT, Al-Jamal KT, Bomans PH, Frederik PM, Kostarelos K Functionalized-quantum-dot-liposome hybrids as multimodal nanoparticles for cancer. Small 2008, 4:1406-1415.

60. Weng KC, Noble CO, Papahadjopoulos-Sternberg B, Chen FF, Drummond DC, Kirpotin DB, Wang D, Hom YK, Hann B, Park JW: Targeted tumor cell internalization and imaging of multifunctional quantum dotconjugated immunoliposomes in vitro and in vivo. Nano Lett 2008, 8:2851-2857.

61. Smith BR, Cheng Z, De A, Koh AL, Sinclair R, Gambhir SS: Real-time intravital imaging of RGD-quantum dot binding to luminal endothelium in mouse tumor neovasculature. Nano Lett 2008, 8:2599-2606. 
62. Chen LD, Liu J, Yu XF, He M, Pei XF, Tang ZY, Wang QQ, Pang DW, Li Y: The biocompatibility of quantum dot probes used for the targeted imaging of hepatocellular carcinoma metastasis. Biomaterials 2008 29:4170-4176.

63. Derfus AM, Chen AA, Min DH, Ruoslahti E, Bhatia SN: Targeted quantum dot conjugates for siRNA delivery. Bioconjug Chem 2007, 18:1391-1396.

64. Voura EB, Jaiswal JK, Mattoussi H, Simon SM: Tracking metastatic tumo cell extravasation with quantum dot nanocrystals and fluorescence emission-scanning microscopy. Nat Med 2004, 10:993-998.

65. lakoubov LZ, Torchilin VP: A novel class of antitumor antibodies: nucleosome-restricted antinuclear autoantibodies (ANA) from healthy aged nonautoimmune mice. Oncol Res 1997, 9:439-446.

66. Hama Y, Koyama Y, Urano Y, Choyke PL, Kobayashi H: Two-color lymphatic mapping using Ig-conjugated near infrared optical probes. J Invest Dermatol 2007, 127:2351-2356.

67. Sampath L, Wang W, Sevick-Muraca EM: Near infrared fluorescent optical imaging for nodal staging. J Biomed Opt 2008, 13:041312.

68. Perini R, Choe R, Yodh AG, Sehgal C, Divgi CR, Rosen MA: Non-invasive assessment of tumor neovasculature: techniques and clinical applications. Cancer Metastasis Rev 2008, 27:615-630

69. Sevick-Muraca EM, Sharma R, Rasmussen JC, Marshall MV, Wendt JA, Pham HQ, Bonefas E, Houston JP, Sampath L, Adams KE, Blanchard DK, Fisher RE, Chiang SB, Elledge R, Mawad ME: Imaging of lymph flow in breast cancer patients after microdose administration of a near-infrared fluorophore: feasibility study. Radiology 2008, 246:734-741.

70. Koyama T, Tsubota A, Nariai K, Mitsunaga M, Yanaga K, Takahashi H: Novel biomedical imaging approach for detection of sentinel nodes in an experimental model of gastric cancer. Br J Surg 2007, 94:996-1001.

71. Niedre MJ, de Kleine RH, Aikawa E, Kirsch DG, Weissleder R, Ntziachristos V: Early photon tomography allows fluorescence detection of lung carcinomas and disease progression in mice in vivo. Proc Natl Acad Sci USA 2008.

72. Koop EJ, van Wees BJ, Reuter D, Wieck AD, van der Wal CH: Spin accumulation and spin relaxation in a large open quantum dot. Phys Rev Lett 2008, 101:056602.

73. Haisch C: Quantitative analysis in medicine using photoacoustic tomography. Anal Bioanal Chem 2009, 393:473-479.

74. Sung Y, Choi W, Fang-Yen C, Badizadegan K, Dasari RR, Feld MS: Optical diffraction tomography for high resolution live cell imaging. Opt Express 2009, 17:266-277.

\section{Pre-publication history}

The pre-publication history for this paper can be accessed here: http://www.biomedcentral.com/1471-2342/10/22/prepub

\section{doi:10.1186/1471-2342-10-22}

Cite this article as: Papagiannaros et al:: Quantum dot loaded immunomicelles for tumor imaging. BMC Medical Imaging 2010 10:22.

\section{Submit your next manuscript to BioMed Central and take full advantage of:}

- Convenient online submission

- Thorough peer review

- No space constraints or color figure charges

- Immediate publication on acceptance

- Inclusion in PubMed, CAS, Scopus and Google Scholar

- Research which is freely available for redistribution

Submit your manuscript at www.biomedcentral.com/submit
Biomed Central 